\title{
Majority of Elderly Sedentary Kenyans Show Unfavorable Body Composition and Cardio-Metabolic Fitness
}

\section{Karani Magutah ${ }^{1,2}$, Nilesh B Patel ${ }^{1}$ and Kihumbu Thairu ${ }^{1}$}

${ }^{1}$ Department of Medical Physiology, University of Nairobi, Nairobi, Kenya

${ }^{2}$ Department of Medical Physiology, Moi University, Eldoret, Kenya

*Corresponding author: Karani Magutah, Department of Medical Physiology, University of Nairobi, Nairobi, Kenya, Tel: +254721545063; E-mail: kmagutah@gmail.com Received date: August 15, 2016, Accepted date: September 07,2016, Published date: September 14,2016

Copyright: @ 2016 Magutah K, et al. This is an open-access article distributed under the terms of the Creative Commons Attribution License, which permits unrestricted use, distribution, and reproduction in any medium, provided the original author and source are credited.

\begin{abstract}
Chronic disease, more prevalent among the elderly, is influenced by body composition and cardio-metabolic physiognomies.

Aim: In this study, body composition, cardiovascular, and metabolic function in sedentary but healthy Kenyan individuals of $\geq 50$ years was determined.

Methods: For 53 volunteers from Eldoret, Kenya, their biological, demographic characteristics, metabolic functions, cardiovascular performance, and body composition measurements were done before they were subjected to a Shuttle Run Test (SRT). Following SRT, their cardiovascular parameters were measured, and maximal oxygen consumption estimated. Fasting blood sugars and lipid profiles were measured after a 12-hour fast.

Results: For males and females, respectively, the following parameters were recorded: average ages $55.5 \pm 3.0$ and $53.9 \pm 3.0$ years, $\mathrm{BMI}>24.9 \mathrm{Kg} / \mathrm{M}^{2}$ in $67 \%$ and $88.5 \%$, waist-to-height ratio $\geq 0.5$ in $70 \%$ and $88.5 \%$, waist-hip ratio $78 \%$ ( $\geq 0.90$ ) and $38.5 \%(\geq 0.85$ ), and blood pressure $>140 / 90 \mathrm{mmHg}$ in $22.2 \%$ and $23.1 \%$. Pre-diabetic to diabetic values were found in $70.4 \%$ males and $88.5 \%$ females. The percent body fat was $22.3 \pm 8.0$ and $38.3 \pm 4.69$ in males and females, respectively. Lipid profile for males and females, respectively, showed high Total Cholesterol (TC) in $48.1 \%$ and $42.3 \%$, anomalous Low Density Lipoproteins (LDL) in $29.6 \%$ and $26.9 \%$, lower than normal HDL levels in $29.6 \%$ and $23.1 \%$, elevated triglycerides in $22.2 \%$ and $7.7 \%$, abnormal TC/HDL ratio in $44.4 \%$ and $38.5 \%$ females, higher LDL/HDL in $33.3 \%$ and $34.6 \%$, and critical Triglycerides/HDL levels in $7.4 \%$ and $3.8 \%$. Overall, $51.9 \%$ of males and $53.8 \%$ of females showed features consistent with metabolic syndrome.
\end{abstract}

Conclusion: Majority of elderly sedentary Kenyans have body compositions and cardio-metabolic profiles below that for good cardiopulmonary fitness.

Keywords: Elderly; Sedentary; Cardio-metabolic fitness; Lipid profiles; Kenyan

\section{Introduction}

Physical inactivity can have an influence on population morbidity and mortality. The World Health Organization (WHO) has identified lack of physical activity as the fourth leading factor contributing to global mortality, and it is expected that it will have more influence with increasing levels of physical inactivity. Apart from contributing to cardiovascular disease, physical inactivity is identified as the key underlying factor in close to a third of all diabetes and ischaemic heart disease events, and up to $25 \%$ of breast and colon cancers [1] Sedentariness is becoming an underrated threat to health in modern times. In Kenya, as economic prosperity increases, many people now drive or ride to work, and sit for many hours at their desks. After work at home, there is limited physical activity as many have house help to do the chores. This lack of physical activity is also compounded by watching TV or playing video games. Currently, preventable noncommunicable diseases account for one third of all mortality in Kenya. In the country, cardiovascular disease is the leading noncommunicable cause of both morbidity and mortality, accounting for
$8 \%$ of deaths across all ages and gender, with diabetes directly contributing for more than $1 \%$ [2]. With rising sedentariness, this may rise in the near future.

The association and effect of lifestyle on body composition and cardio-metabolic features is more evident amongst older individuals [3]. With the current average life expectancy in Kenya at 58 years; the maximal lifespans being 57 and 59 years for males and female respectively, a 50 year old Kenyan may be considered elderly [4]. While WHO puts the chronological age for elderly at 65 years for developed countries, agreement that life-expectancy differences and regional social-economic factors that affect mortality may affect this definition for Africa allows for adoption of lower cut-offs for the Sub-Saharan Africa (UN, 2012), a region belabored by lower life expectancies.

After 50 years of age, the link between higher body mass index and increased mortality and morbidity outcomes has been demonstrated [3,5]. Central obesity above 0.5 , calculated by waist-to-hip ratio, also positively correlates with increasing morbidity and mortality [6-11]. In addition, waist to height cut-off points has also been shown to be useful for assessing metabolic risks and complications [12]. Skin fold measurements from specific body areas have been used to develop equations for predicting body density for both men and women 
[13-15], which can then be used to estimate body fat percentage [16] These simple and non-invasive approaches are suitable in resource constrained areas where it is not possible to employ the complex and expensive under-water-weighing, a gold standard for body composition determination [17].

Cardio-metabolic characteristics are also used to assess disease risk across all ages. Reference cut-offs have been published for blood lipid profiles and ratios for unfavorable health risks associated with poor cardiorespiratory fitness and cardiovascular disease [18-20]. Other physiognomies for estimation of metabolic risks and disease, for which cut off points are known, include fasting blood sugar, blood pressure (BP) and pulse rates (HR) [12,21]. From these parameters, it is possible to classify the disease risk of an individual and plan or advice on appropriate preventive and control measures.

With chronic diseases now becoming a larger burden among elderly Kenyans, and, further, with Kenyans adopting more sedentary lifestyles, this study was undertaken to measure cardio-respiratory function, lipid profiles and glucose levels in apparently healthy, elderly Kenyans.

\section{Materials and Methods}

Fifty-three (27 males and 26 females) healthy sedentary participants aged $\geq 50$ years from Eldoret, Uasin Gishu County, Kenya, were purposively recruited through local advertisement in the print media. Sedentary individuals were identified as those not meeting the 600 metabolic equivalents (MET) minutes per week threshold using the WHO Global Physical Activity Questionnaire (GPAQ) analysis. Volunteers with cardio-respiratory, physical or other ailments and those confirming recent use of $\beta$-blockers and related drugs were excluded [22]. Further, a qualified first-aider was available during the exertion stage of the protocol. Study approval was obtained from the Moi Teaching and Referral Hospital /Moi University Institutional Research Ethics Committee and reproducibility was enhanced by having the same researcher conduct all measurements on the multiple participants for consistency.

Upon signed consent from participants, a form detailing their biodemographic characteristics and exercise/physical activity patterns was initially filled out. For each participant, height and weight without shoes but on light clothing was measured using a stadiometer and a mechanical scale (CAMRY Mechanical scale, BR9012, Shanghai, China). Waist and hip circumference was measured with a tape measure. Skin fold measures, using calipers (Harpenden Skinfold Calipers, BATY International, England), were taken from three different body sites: chest, abdomen, and thigh for males, and the triceps, supra-iliac, and thigh for females. From these measurements, BMI, waist-to-hip ratio, and waist-to-height ratio were calculated. The sum of the 3 skin fold measurements were used to determine Body Density (BD) using published generalized equations [13-15], while the fat percentage was computed from body densities using Brozek formulae [16].

The $20 \mathrm{M}$ shuttle run test (SRT) was used for maximal oxygen uptake $\left(\mathrm{VO}_{2 \max }\right)$ estimation. It is a non-invasive, affordable yet more practicable field test for energy expenditure assessment that could estimate aerobic fitness in resource constrained environments. Its scoring is based on the highest level attained in the 21-level protocol that entails incremental running speeds by utilizing pre-recorded sound signals [23]. This ensures a gradual increase in work rate and thus the exercise intensity by having subjects run to exhaustion while maintaining cadence with the sound signals. The SRT has been validated to be completed on flat indoor surfaces using workloads lasting approximately one minute in each of its levels [24]. It is an accurate multi-stage test of cardiovascular fitness and $\mathrm{VO} 2 \mathrm{max}$ estimation, by exercise intensity applications.

Before the SRT, BP and HR were measured with an electronic sphygmomanometer (Omron ${ }^{\circledR}$ M2 (HEM 7119E)) and then at exhaustion from the SRT, and over the next 5 minutes rest to follow BP normalization. The level of performance reached was used to estimate $\mathrm{VO}_{2 \max }$ [23]. The standard provided by the American College of Sports Medicine (ACSM) and the Cooper Institute for Aerobics Research was used for interpretation of the $\mathrm{VO}_{2 \max }$ [25].

Venous blood samples were collected after 12 hours of overnight fasting and blood glucose determined using a glucometer (Freestyle Optium Abbottcentrifugation within an hour of collection, and the serum was frozen at $-12^{\circ} \mathrm{C}$ until lipid profile assays were done, usually at the end of each week, by Academic Model Providing Access to Health care (AMPATH) reference laboratories in Eldoret, Kenya. Basic lipid profiles: Total Cholesterols (TC), High Density Lipoproteins (HDL), Low Density Lipoproteins (LDL), and Triglycerides (TG) were determined and the related ratios of TC/HDL, LDL/HDL and TG/HDL calculated and compared with published cut-off points.

Data were analysed with STATA version 13. Summary statistics and t-test functions were used, and means, standard deviations, and their related ranges were the outputs. $\mathrm{P}$ value for significance of comparisons was set at $\leq 0.05$.

\section{Results}

The mean age of the males $(\mathrm{n}=27)$ was $55.52 \pm 3.02$ years and 53.88 \pm 3.0 years for the females $(n=26)$. Tertiary level of education was achieved by $88.4 \%$ of males and $70.4 \%$ of females, the rest had secondary level education. Their ethnicities and occupations were diverse, and they were either in white collar jobs $(9.4 \%)$ or retired (90.6\%) from white collar jobs.

In $66.7 \%$ of males and $88.5 \%$ of females, BMI was above 24.9 $\mathrm{Kg} / \mathrm{M}^{2}$. Waist-to-height cut-off ratios above 0.5 were seen in $70.4 \%$ of males $(0.54 \pm 0.07)$ and $88.5 \%$ of females $(0.58 \pm 0.75)$. Waist-hip ratios of males and female averaged $0.94 \pm 0.06 \mathrm{~cm}$ and $0.82 \pm 0.11 \mathrm{~cm}$, respectively, with $78 \%$ and $38.5 \%$ of male and female having ratios $\geq$ 0.90 and $\geq 0.85$ respectively.

Pre-SRT test blood pressure $\geq 140 / 90 \mathrm{mmHg}$ was measured in $22.2 \%$ males and $23.1 \%$ females. In addition, those with systolic pressure above $140 \mathrm{mmHg}$ and diastolic pressure below $90 \mathrm{mmHg}$ were $14.8 \%$ and $15.4 \%$ for males and female, respectively. Only one each of the males and females had diastolic pressure above $90 \mathrm{mmHg}$ and systolic pressure below $140 \mathrm{mmHg}$. The pre- and 5 minutes post SRT blood pressures were not statistically significant (systolic pressure: $\mathrm{p}=0.12$ and $\mathrm{p}=0.26$; diastolic pressure: $\mathrm{p}=0.83$ and $\mathrm{p}=032$ for males and females, respectively).

The baseline mean fasting blood glucose levels was $5.92 \pm 1.4$ $\mathrm{mmol} / \mathrm{l}$ for males and $6.23 \pm 0.74 \mathrm{mmol} / \mathrm{l}$ for females. Glucose levels above $5.5 \mathrm{mmol} / \mathrm{l}$ indicative of pre-diabetic to diabetic levels, were found in $70.4 \%$ males and $88.5 \%$ females.

Using the generalized equations for prediction of body density (BD) for men $(\mathrm{BD}=1.10938-(0.0008267 \mathrm{x}$ sum of chest, abdomen, and thigh skinfolds in $\mathrm{mm})+(0.0000016 \mathrm{x}$ square of the sum of chest, 
Page 3 of 6

abdomen and thigh $)-(0.0002574 \mathrm{x}$ age $))$ and women $(\mathrm{BD}=1.0994921$ - (0.0009929 x sum of triceps, thigh, and supra iliac skinfolds $)+$ $(0.0000023 \times$ square of the sum of triceps, thigh and supra iliac skinfolds) - (0.0001392 $\mathrm{x}$ age) [13-15], the male body density was 1.05 \pm 0.19 and, for females, it was $1.01 \pm 0.1$. The calculated percent body fat from body density, using Brozek formula: body fat $=(4.57 / \rho-$ $4.142) \times 100$, was $22.26 \pm 7.95 \%$ (males) and $38.33 \pm 4.69 \%$ (females).

For blood chemistry, $48.1 \%$ of males and $42.3 \%$ of females had baseline TC levels above the cut-off of 5.2 and $5.3 \mathrm{mmol} / \mathrm{l}$, respectively. In $29.6 \%$ of males and $26.9 \%$ of females, LDL values were outside the 1.3-3.9 mmol/l range. A similar percentage (29.6\%) of males and $23.1 \%$ of females had HDL levels $<0.9 \mathrm{mmol} / \mathrm{l}$ while $22.2 \%$ of males and $7.7 \%$ of females had TG levels above $2.3 \mathrm{mmol} / \mathrm{l}$.

TC/HDL ratios above 5.0 and 4.5 were found in $44.4 \%$ of males and $38.5 \%$ of females, respectively. The mean TC/HDL ratio for males above cut-off was $6.61 \pm 1.19$ (range 5.23-8.97) and for females it was $5.40 \pm 0.73$ (range 4.64-6.96). Thirty three percent of males had LDL/HDL values above $3.5(4.47 \pm 0.87)$ and $34.6 \%$ of females had LDL/HDL values above $3(3.46 \pm 0.32)$. Males and female with TG/HDL above 3.5 and 2.5 were $7.4 \%$ and $3.8 \%$, respectively, with means of $4.07 \pm 2.01$, and $2.78 \pm 0$. The rest of the demographic and clinical characteristics of the participants are shown in Table 1.

\section{Discussion}

In this study, we found that males were overweight while females were in the obese class I, which has long been associated with unfavorable health outcomes [26-28]. The high BMI of the participants in the study suggests that they are at an increased risk for cardiovascular disease and associated metabolic syndrome. A few meta-analyses and cohort studies, however, have proposed the very low and high values outside the $23-33 \mathrm{~kg} / \mathrm{m}^{2}$ range as increasing the mortality risks among the elderly, so that higher BMI values as observed in the current study may actually be associated with better cardiovascular disease epidemiology in this cohort [29-32].

Recent studies have advanced the argument that waist-to-height ratio is an important anthropometric measure for health, and is superior to traditional measures such as BMI, in assessing cardiovascular and metabolic risk among different races and ages $[6-8,10,33,34]$. All participants in the current study had mean waist-toheight ratios above the 0.5 cut-off, which has been shown to pose higher health risks [6,8-10]. In fact, considering the argument that this ratio is superior to BMI in assessing health risks and outcomes amongst apparently healthy populations as it particularly considers fat distribution $[7,33,35]$, the participants in this study are at a higher risk of metabolic syndrome.

\begin{tabular}{|l|l|l|}
\hline & Male $(\mathrm{n}=27)$ & Female $(\mathrm{n}=26)$ \\
\hline Age (years) & $55.52 \pm 3.02$ & $53.88 \pm 3$ \\
\hline BMI $\left(\mathrm{kg} / \mathrm{m}^{2}\right)$ & $27.13 \pm 4.55$ & $32.32 \pm 5.07$ \\
\hline Waist/Height Ratio & $0.54 \pm 0.07$ & $0.58 \pm 0.75$ \\
\hline Waist/Hip Ratio & $0.94 \pm 0.06$ & $0.82 \pm 0.11$ \\
\hline Sum of 3 skinfolds $(\mathrm{mm})$ & $68.76 \pm 30.85$ & $113.81 \pm 23.31$ \\
\hline Body density & $1.05 \pm 0.19$ & $1.01 \pm 0.1$ \\
\hline Fat\% & $22.26 \pm 7.95$ & $38.33 \pm 4.69$ \\
\hline
\end{tabular}

\begin{tabular}{|l|l|l|}
\hline Pre-exertion Systolic BP $(\mathrm{mmHg})$ & $139.81 \pm 19.42$ & $135.12 \pm 19.83$ \\
\hline Pre-exertion Diastolic BP $(\mathrm{mmHg})$ & $82.93 \pm 9.9$ & $83.77 \pm 9.53$ \\
\hline Pre-exertion heart rate $(\mathrm{b} / \mathrm{m})$ & $75.3 \pm 8.64$ & $75.77 \pm 10.93$ \\
\hline Exhaustion Systolic BP $(\mathrm{mmHg})$ & $194.04 \pm 18.75$ & $189.73 \pm 25.4$ \\
\hline Exhaustion Diastolic BP $(\mathrm{mmHg})$ & $83.52 \pm 9.48$ & $84.42 \pm 10.18$ \\
\hline Exhaustion heart rate $(\mathrm{b} / \mathrm{m})$ & $126.44 \pm 13.86$ & $124.12 \pm 15.38$ \\
\hline 5 min post-exertion Systolic BP $(\mathrm{mmHg})$ & $135.44 \pm 15.09$ & $127.73 \pm 14.48$ \\
\hline 5 min post-exertion Diastolic BP $(\mathrm{mmHg})$ & $77.37 \pm 9.34$ & $75.08 \pm 10.49$ \\
\hline 5 min post-exertion heart rate $(\mathrm{b} / \mathrm{m})$ & $90.81 \pm 10$ & $88.27 \pm 9.85$ \\
\hline VO ${ }_{2 m a x}(\mathrm{ml} / \mathrm{kg} / \mathrm{min})$ & $25.39 \pm 4.44$ & $20.36 \pm 1.93$ \\
\hline Time in SRT (Seconds) & $176.04 \pm 80.33$ & $85.08 \pm 32.96$ \\
\hline Fasting blood glucose $(\mathrm{mmol} / \mathrm{l})$ & $5.92 \pm 1.4$ & $6.23 \pm 0.74$ \\
\hline Total Cholesterol $(\mathrm{TC})(\mathrm{mmol} / \mathrm{l})$ & $5.19 \pm 1.52$ & $5.26 \pm 1.94$ \\
\hline High density lipoproteins $(\mathrm{HDL})(\mathrm{mmol} / \mathrm{l})$ & $1.18 \pm 0.41$ & $1.19 \pm 0.34$ \\
\hline Low density lipoproteins $(\mathrm{LDL})(\mathrm{mmol} / \mathrm{l})$ & $3.15 \pm 1.05$ & $3.14 \pm 1.03$ \\
\hline Triglycerides (TG) $(\mathrm{mmol} / \mathrm{l})$ & $1.81 \pm 1.09$ & $1.25 \pm 0.76$ \\
\hline TC/HDL ratio & $4.84 \pm 2$ & $4.41 \pm 0.97$ \\
\hline LDL/HDL ratio & $2.96 \pm 1.34$ & $2.67 \pm 0.69$ \\
\hline TG/HDL ratio & $1.91 \pm 1.69$ & $1.1 \pm 0.62$ \\
\hline Note: Data presented as means $\pm \mathrm{standard}$ & deviation. & \\
\hline
\end{tabular}

Table 1: Demographic and clinical characteristics of the elderly $(\geq 50$ years) Kenyans.

A comparison of the waist-hip ratio against WHO cut-off guidelines of $\geq 0.90$ (male) and $\geq 0.85$ (female) [12], associated with substantially increased metabolic complications [12], showed that around $80 \%$ of the male participants had central/abdominal obesity with mean value above the WHO cut-off, as compared to less than $40 \%$ of the females. This indicates that males in this study have a relatively higher risk for metabolic syndrome and other health issues [28]. Females have half the risk of their male counterparts.

Determination of the basic lipid profiles is one approach to assess cardiorespiratory fitness amongst apparently healthy individuals and those with metabolic syndrome diagnoses. However, the use of the different lipid ratios is now more common. These ratios are now recognized to be independently associated with cardiovascular disease risks $[18,19]$. When published cut-offs for unfavorable risk categories associated with poor cardiorespiratory fitness and cardiovascular disease were considered [18-20], $44 \%$ of the males and $39 \%$ of the females had values above the cut-offs for TC/HDL baseline ratios (above 5.0 and 4.5, respectively) suggesting that these individuals are at substantial metabolic risks. Similarly, with 3.5 and 3.0 cut-offs for LDL/HDL for males and females respectively, 33\% of males and $35 \%$ of females in this study had higher values, and with the absolute means far above the cut-offs. While this alone also shows high health risks, combination of these two ratios suggests enhanced metabolic syndrome risk for this elderly population. Separately, taking TG/HDL cut-offs of 3.5 and 2.5 for males and females, respectively, $7 \%$ of the 
males and $4 \%$ of the females in this study showed higher risk of cardiometabolic disorder. However, the available TG/HDL cut-offs have only been proposed for European populations [20]. There are no comparable studies for black populations to assess if racial differences might exist.

Assessment of the basic lipid profiles showed that nearly half of the participants had total cholesterol (TC) levels above the reference cutoff for their respective gender, namely 5.2 and $5.3 \mathrm{mmol} / \mathrm{l}$ for males and females, respectively. Nearly half the participants are at a high risk of cardiovascular disease, since absolute TC level is independently associated with this disorder, unless they undertake interventions to reduce their TC levels. For LDL values, close to one third of the participants had unfavorable values, and similarly, negative values for HDL levels, below the standard reference of $<0.9 \mathrm{mmol} / \mathrm{l}$. Furthermore, with a large percentage of participants having high triglyceride levels, all this points to a population with health risks. In particular, using lipid profiles alone suggests that more Kenyan elderly males as opposed to elderly females are at risk, which is in agreement with a previous study from a Kenyan rural setting [36].

Body composition reference data for the elderly African population is unavailable. While data abounds elsewhere, it is based on nonAfrican populations, and cause comparison difficulties where racial differences may be present. For instance, while males in the current study had fat mass of $22 \%$ and their female counterparts a fat mass of $38 \%$, an Indian population had percentages of 25 and 30, respectively, though this covered a wider age-range [37]. A 15-year bi-annual crosssectional study to determine cut-offs for a US population found higher percentages for women, but the categorization was based on the various BMI subsets used for analysis, and therefore not entirely comparable with the current study [38]. The high percentage body fat for the female in the current study, making well over one third of their body mass, and with a concurrent lower body density, is consistent with the blood chemistry findings where the females had higher total cholesterol values. Based on Jackson and Pollock equations [11-13], males in this study have average body fat but their female counterparts are obese.

In this study, participants showed impaired fasting blood glucose levels. Both genders had pre-diabetic mean glucose values, a finding which is consistent with other studies that reported elevated fasting levels of plasma glucose with age in other African populations $[39,40]$. However, in this study females had higher means than their agematched males. Results from studies of urban Nigerians and rural Kenyans differ [36,41], and part of the difference may be due to the relatively younger populations used in the two studies.

The mean pre-exertion systolic and diastolic BP was within the normal ranges, i.e., below 140 and $90 \mathrm{mmHg}$, respectively. Further, the systolic BP (SBP) rise and associated diastolic BP (DBP) decay observed at exhaustion from the SRT, and the return to normal ranges within the following five minutes of rest was within the expected published values [42]. The 5-minutes post SRT blood pressure was similar to the pressure before the run test portraying good normalization. However, as nearly a quarter of the participants had pre-exertion BP above 140/90, this indicates that a significant proportion of the participants have hypertensive tendency.

Based on reference VO2max values provided by the American College of Sports Medicine [25], the mean values for the males and females in this study were below values expected for their ages. Both males and female spent much less time in the SRT than expected for their age, which translated to lower VO2max. These showed reduced cardiorespiratory health consistent with their sedentary life style.

Using Alberti criterion for metabolic syndrome diagnosis, where at least three of the following criterion have to present: waist circumference above 102 and $88 \mathrm{~cm}$ for males and females, respectively. $\mathrm{HDL}<1.0$ and $<1.3 \mathrm{mmol} / \mathrm{l}$ in males and females, respectively, TG $\geq 1.7 \mathrm{mmol} / \mathrm{l}, \mathrm{SBP} \geq 130 \mathrm{mmHg}$ or $\mathrm{DBP} \geq 85 \mathrm{mmHg}$, and fasting blood glucose $>5.6 \mathrm{mmol} / \mathrm{l}[12,21]$, a high percentage of participants in this study showed unfavorable health characteristics. Well above half the males and females showed at least three of the criterions for a positive metabolic syndrome diagnosis and appear to have some form or grade of this syndrome. The current findings indicate a potentially poor future health outcomes for this cohort as it is likely that the measured clinical features will get worse with their advancing age and associated sedentariness $[43,44]$. The findings of this study are consistent with other studies done in the East African region, which showed increasing prevalence of risk factors for cardiovascular disease and metabolic syndrome [45].

\section{Limitations}

The current study used 3-point skin fold measures' method of body fat composition estimation, whose accuracy is subjective by being dependent on the observer. This may affect reproducibility of results [46]. In the current study, this was overcome by having the same researcher conduct all measurements on the multiple participants for consistency.

\section{Conclusion}

In this study, the bio-demographic and clinical characteristics of participants were found to be associated with reduced cardiopulmonary fitness, an issue that needs to be addressed to improve the future health outcomes of this elderly and sedentary population.

\section{Author Contributions}

Karani Magutah (MPH,MSc) helped in designing protocol, data collection, analysis and writing. Kihumbu Thairu (PhD) and Nilesh Patel (PhD) helped with designing protocol and writing the manuscript.

\section{Declaration of Conflict of Interest}

Authors declare no conflict of interest regarding the research work and its outcomes

\section{Funding}

This research was supported by the Consortium for Advanced Research Training in Africa (CARTA). CARTA is jointly led by the African Population and Health Research Center (APHRC) and the University of the Witwatersrand and funded by the Wellcome Trust (UK) (Grant No: 087547/Z/08/Z), the Department for International Development (DfID) under the Development Partnerships in Higher Education (DelPHE), the Carnegie Corporation of New York (Grant No: B 8606), the Ford Foundation (Grant No: 1100-0399), Google.Org (Grant No: 191994), Sida (Grant No: 54100029), MacArthur Foundation (Grant No: 10-95915-000-INP) and British Council. 


\section{References}

1. World Health Organization (2010) Global Recommendations on Physical Activity for Health.

2. World Health Organization (2014)Noncommunicable Diseases (NCD) Country Profiles.

3. Bea JW (2015) Risk of Mortality According to Body Mass Index and Body Composition Among Postmenopausal Women. Am J Epidemiol 182: 585-596.

4. Kenya National Bureau of Statistics (2015) National AIDS Control Council, Kenya Medical Research Institute, National Council for Population and Development, Nairobi, Kenya, and The DHS Program, ICF International, Rockville, Maryland, USA Kenya Demographic and Health Survey 2014.

5. Adams KF (2006) Overweight, obesity, and mortality in a large prospective cohort of persons 50 to 71 years old. $\mathrm{N}$ Engl J Med 355:763-778.

6. Browning LM, Hsieh SD, Ashwell M (2010) A systematic review of waistto-height ratio as a screening tool for the prediction of cardiovascular disease and diabetes: 0.5 could be a suitable global boundary value. Nutr Res Rev 23: 247-269.

7. Ashwell M, Gunn P, Gibson S (2006) Waist-to-height ratio is a better screening tool than waist circumference and BMI for adult cardiometabolic risk factors: systematic review and meta-analysis. Obes Rev13: 275-286.

8. Ashwell M, Gibson S (2014) A proposal for a primary screening tool: 'Keep your waist circumference to less than half your height'. BMC Med 12: 207.

9. Ashwell M, Cole TJ, Dixon AK (1996) Ratio of waist circumference to height is strong predictor of intra-abdominal fat. Bmj 313: 559-560.

10. Ashwell M (2012) Plea for simplicity: use of waist-to-height ratio as a primary screening tool to assess cardiometabolic risk. Clin Obes 2: 3-5.

11. Joseph B (2015) Hips don't lie: Waist-to-hip ratio in trauma patients. J Trauma Acute Care Surg 79: 1055-1061.

12. WHO (2008) Waist circumference and waist-hip ratio: Report of a WHO expert consultation, Geneva 8-11.

13. Jackson AS, Pollock ML (2004) Generalized equations for predicting body density of men. 1978. Br J Nutr 91: 161-168.

14. Jackson AS, Pollock ML, Ward A (1980) Generalized equations for predicting body density of women. Med Sci Sports Exerc 12: 175-181.

15. Jackson AS, Pollock ML (1978) Generalized equations for predicting body density of men. Br J Nutr 40: 497-504.

16. Brozek J (1963) Densitometric analysis of body composition: Revision of some quantitative assumptions. Ann N y Acad Sci 110:113-140

17. Shen W, Chen J (2008) Application of imaging and other noninvasive techniques in determining adipose tissue mass. Methods Mol Biol 456: 39-54.

18. Gotto AM, Assmann G, Carmena R (2000) The ILIB lipid handbook for clinical practice: blood lipids and coronary heart disease. 2nd ed. New York.International Lipid Information Bureau 201: 52-53.

19. Millan J (2009) Lipoprotein ratios: Physiological significance and clinical usefulness in cardiovascular prevention. Vasc Health Risk Manag 5 757-765.

20. Salazar MR (2012) Relation among the plasma triglyceride/high-density lipoprotein cholesterol concentration ratio, insulin resistance, and associated cardio-metabolic risk factors in men and women. Am J Cardiol 109: 1749-1753.

21. Alberti KG (2009) Harmonizing the metabolic syndrome: a joint interim statement of the International Diabetes Federation Task Force on Epidemiology and Prevention; National Heart, Lung, and Blood Institute; American Heart Association; World Heart Federation; International Atherosclerosis Society; and International Association for the Study of Obesity. Circulation 120: 1640-1645.
22. Joyner MJ (1985) Effects of beta-blockade on exercise capacity of trained and untrained men: a hemodynamic comparison. J Appl Physiol 60: 1429-1434.

23. Ramsbottom R, Brewer J, Williams C (1988) A progressive shuttle run test to estimate maximal oxygen uptake. Br J Sports Med 22: 141-144.

24. Leger LA, Lambert J (1982) A maximal multistage 20-m shuttle run test to predict VO2 max. Eur J Appl Physiol Occup Physiol 49: 1-12.

25. Heyward HV (1988) The Physical Fitness Specialist Certification Manual, The Cooper Institute for Aerobics Research. Dallas TX 3rd Ediition ed.

26. WHO (1995) Physical status: the use and interpretation of anthropometry. Report of a WHO Expert Committee. WHO Technical Report Series 854. Geneva: World Health Organization.

27. Berrington de GonzalezA (2010) Body-mass index and mortality among 1.46 million white adults. N Engl J Med 363: 2211-2219.

28. Myint PK (2014) Body fat percentage, body mass index and waist-to-hip ratio as predictors of mortality and cardiovascular disease. Heart 100: 1613-1619.

29. Winter JE (2014) BMI and all-cause mortality in older adults: a metaanalysis. Am J Clin Nutr 99: 875-890.

30. Veronese $\mathrm{N}$ (2015) Inverse relationship between body mass index and mortality in older nursing home residents: a meta-analysis of 19,538 elderly subjects. Obes Rev 16: 1001-1015.

31. Ahmadi SF (2015) Reverse Epidemiology of Traditional Cardiovascular Risk Factors in the Geriatric Population. J Am Med Dir Assoc 16: 933-939.

32. Wu CY (2014) Association of body mass index with all-cause and cardiovascular disease mortality in the elderly. PLoS One 9: e102589.

33. Hsieh SD, Muto T (2005) The superiority of waist-to-height ratio as an anthropometric index to evaluate clustering of coronary risk factors among non-obese men and women. Prev Med 40: 216-220.

34. Savva SC, Lamnisos D, Kafatos AG (2013) Predicting cardiometabolic risk: waist-to-height ratio or BMI. A meta-analysis. Diabetes Metab Syndr Obes 6: 403-419.

35. Zhu Q (2014) Waist-to-height ratio is an appropriate index for identifying cardiometabolic risk in Chinese individuals with normal body mass index and waist circumference. J Diabetes 6: 527-534.

36. Christensen DL (2015) cardiovascular risk factors in rural Kenyans are associated with differential age gradients, but not modified by sex or ethnicity. Ann Hum Biol 1-8.

37. Marwaha RK (204) normative data of body fat mass and its distribution as assessed by DXA in Indian adult population. J Clin Densitom 17: 136-142.

38. Heo M (2012) Percentage of body fat cutoffs by sex, age, and raceethnicity in the US adult population from NHANES 1999-2004.Am J Clin Nutr 95: 594-602.

39. Alikor CA, Emem-Chioma PC (2015) Epidemiology Of Diabetes And Impaired Fasting Glucose In A Rural Community Of Nigerian Niger Delta Region. Niger J Med 24: 114-124.

40. Werfalli M (2015) The prevalence of type 2 diabetes among older people in Africa: a systematic review. Lancet Diabetes Endocrinol 4:72-84

41. Sabir AA, Isezuo SA, Ohwovoriole AE (2011) Dysglycaemia and its risk factors in an urban Fulani population of northern Nigeria. West Afr J Med 30: 325-330.

42. Nonogi H (1988) Diastolic properties of the normal left ventricle during supine exercise. Br Heart J 60: 30-38.

43. Jackson AS (2009) Role of lifestyle and aging on the longitudinal change in cardiorespiratory fitness. Arch Intern Med 169: 1781-1787.

44. Lakka TA (2003) Sedentary lifestyle, poor cardiorespiratory fitness, and the metabolic syndrome. Med Sci Sports Exerc 35: 1279-1286.

45. Njelekela MA (2009) Gender-related differences in the prevalence of cardiovascular disease risk factors and their correlates in urban Tanzania. BMC Cardiovasc Disord 9: 30.

46. Pollock ML, Jackson AS (1984) Research progress in validation of clinical methods of assessing body composition. Med Sci Sports Exerc 16: 606-615. 